\title{
Laryngeal and Vocal Characterization of Asymptomatic Adults With Sulcus Vocalis
}

\author{
Alex Bruno Soares ${ }^{10}$ Bruno Teixeira de Moares ${ }^{1}$ \\ Noemi Grigoletto de Biase ${ }^{3}$ Jonia Alves Lucena ${ }^{2}$ \\ ${ }^{1}$ Department of Otorhinolaringology, Health Sciences Center, \\ Universidade Federal de Pernambuco, Recife, PE, Brazil \\ 2 Department of Fonoaudiology, Health Sciences Center, \\ Universidade Federal de Pernambuco, Recife, PE, Brazil \\ 3 Paulista Medical School, Department of Otorhinolayngology \\ and Head and Neck Surgery, Universidade Federal de São Paulo, \\ São Paulo, SP, Brazil
}

Ana Nery Barbosa de Araújo ${ }^{2}$

Int Arch Otorhinolaryngol 2019;23:e331-e337.

\begin{abstract}
Address for correspondence Alex Bruno Soares, MD, MSc, Rua Arnado de Albuquerque, 501, apto 1301, Lauritzen, Campina Grande, Paraíba, PB, 58401-390, Brazil

(e-mail: alex.orl@hotmail.com).
\end{abstract}

\begin{abstract}
Keywords

- voice

- vocal cords

- voice quality

Introduction Sulcus vocalis is defined as a longitudinal depression on the vocal cord, parallel to its free border. Its most marked characteristic is breathlessness, caused by incomplete glottal closure, in addition to roughness, due to the decrease in mucosal wave amplitude of the vocal cords. Vocal acoustic aspects, such as fundamental voice frequency, jitter, and shimmer, may also be altered in individuals with this type of laryngeal disorder. To assess the voice of individuals with sulcus vocalis, studies generally include a sample of subjects with vocal symptoms, excluding asymptomatic persons. To better characterize the vocal characteristics of individuals with sulcus vocalis, their asymptomatic counterparts must also be included.

Objective Characterize the larynx and voice of asymptomatic adults with sulcus vocalis. Method A total of 26 adults, 13 with sulcus vocalis (experimental group) and 13 without (control group) were assessed. All the participants were submitted to suspension microlaryngoscopy, voice self-assessment, auditory perception and acoustic evaluation of the voice.

Results Among the individuals with sulcus vocalis, $78 \%$ of the sulci were type I and $22 \%$ type II. Auditory perception assessment obtained statistically significant lower scores in individuals with sulcus vocalis compared with the control group, and a slight difference in the overall degree of hoarseness and roughness. No statistically significant intergroup diferences were found in self-reported voice or acoustic assessment.

Conclusion Type I was the predominant sulcus vocalis observed in individuals without voice complaints, who may also exhibit slight changes in vocal quality and roughness.
\end{abstract}

\section{Introduction}

Several factors can interfere in vocal quality, including biological, psychological or socioeducational aspects. ${ }^{1}$ However, the

(1D)Alex Bruno Soares's ORCID is https://orcid.org/0000-0001-58351514.

received

October 9, 2018

accepted

March 17, 2019 
conformation seems not to be found in the entire population. ${ }^{3}$ Small anatomical alterations in the larynx, such as the sulcus vocalis, can change its functional result, predisposing individuals to dysphonia (hoarseness) or vocal fatigue. .,2,4 $^{1,4}$

The sulcus vocalis is defined as a longitudinal depression in a vocal cord parallel to its free border, which can vary in extension and depth, and be unilateral or present in both vocal cords. Histologically, the sulcus is located on the surface layer of the lamina propria and is lined with the stratified epithelium, contiguous to the epithelium with a normal mucosal lining. ${ }^{2}$ Sulcus changes are classified according to their morphological characteristics and the degree to which the vocal cord structures are compromised. Ford et al $(1996)^{5}$ divided sulcus disorders of the vocal folds into 3 groups: In type I, epitelial invagination is limited to the lamina propria; type II, epithelial invagination along the vocal fold length; type III is the true sulcus vocalis (pocket type) and represents an epithelial invagination that may penetrate into the vocal ligament and/or vocalis muscle layers. Pontes et al $(1994)^{2}$ propose the following categories: sulcus stria minor-epithelial invagination, whose upper and lower lips usually touch each other; sulcus stria majorspindle-shaped mucosal depression, with a stiffer consistency and adhering to deeper structures, such as the vocal ligament and muscle; pouch-shaped sulcus-lesion that emerges as an invagination, whereby its lips touch each other and the opening leads to a dilated pouch-shaped subepithelial space.

The real incidence of sulcus vocalis is unknown, due to three factors: lack of knowledge of this laryngeal alteration, diagnostic error, or the absence of diagnosis when vocal symptoms are not serious enough to cause vocal complaints. ${ }^{6}$ Currently, examinations such as videolaryngoscopy, videolaryngostroboscopy or suspension microlaryngoscopy are used to investigate morphological and structural changes in the vocal cords, although it is important to consider the data related to the clinical history of vocal alterations. ${ }^{1,6-9}$

It is important to underscore that the sulcus vocalis is not always evident in videolaryngoscopy and often causes only slight structural alterations, although the vocal repercussions can be considerable. ${ }^{2,6-8}$ Videolaryngostroboscopy can help assess a larynx with sulcus vocalis, showing a decline or absence of mucosal wave vibration. ${ }^{1,9}$ However, under some circumstances, an accurate diagnosis of the sulcus vocalis can only be obtained by suspension microlaryngoscopy, the gold standard for diagnosing minimal structural changes. It is applied exceptionally because of its invasive nature and the fact that the procedure is performed under general anesthesia. ${ }^{8,9}$ Suspension microlaryngoscopy makes it possible to assess vocal cord details under binocular microscopy at depth and with good lighting, enabling the use of instruments for palpating vocal cord alterations and providing an important contribution to sulcus vocalis diagnosis. ${ }^{8,9}$

With respect to characterizing visual laryngeal, auditory perception and acoustic attributes, studies performed with symptomatic individuals show that most vocal sulci are bilateral, with types II and III being the most common. ${ }^{5,10-12}$ The most marked vocal characteristic of this lesion is breathless- ness, which results from incomplete glottal closure. Another vocal parameter is roughness, due to the decline in mucosal wave vibration in the vocal cords. ${ }^{1,2,7,12}$ In regard to the acoustic characteristics of voice, parameters such as fundamental voice frequency, jitter, and shimmer were altered. ${ }^{1,12}$

However, it is important to emphasize that voice assessment studies in individuals with sulcus vocalis ${ }^{5,9-15}$ generally select a symptomatic population, excluding possible subjects with sulcus that did not display voice symptoms. As such, the aim of the present study was to characterize the larynx and voice of asymptomatic adults with sulcus vocalis from the standpoint of laryngeal, auditory perception, and acoustic assessment, in addition to voice self-evaluation.

\section{Method}

This is a cross-sectional observational study, conducted in the otolaryngology department of a public hospital in Pernambuco state, Brazil.

After approval was obtained from the institutional research committee, under protocol number 973.637, and the subjects gave their informed consent, data collection occurred between January and December 2014.

The initial sample, selected consecutively by convenience, consisted of 77 adults with no vocal complaints, submitted to general anasthesia for surgery at the extralaryngeal site, extraneous to the study. The subjects were submitted to the following surgeries: tonsillectomies, septoplasties, turbinectomies and/or sinusectomies. After exclusion criteria were applied, the number of patients declined to 71 . Suspension microlaryngoscopy was conducted, revealing 13 individuals with sulcus vocalis (group 1). Among the remaining subjects, 13 gendermatched controls with no laryngeal alterations were selected consecutively (group 2), totaling 26 study participants. Each group consisted of nine women and four men.

Excluded from the study were patients submitted to surgery with high anesthetic risk (above ASA III); endotracheal intubation or previous laryngeal surgery; history of cervical trauma; extrinsic laryngeal aggression factors, including the prolonged use of inhalatory costocorticoids, smoking and occupational respiratory diseases, contraindication for suspension laryngoscopy; trauma from orotracheal intubation; presence of phonotraumatic lesions identified during the examination; and incomplete laryngeal exposure during the procedure.

Suspension microlaryngoscopy was conducted by an otalaryngologist (larynx specialist) using a Zeiss microscope equipped with a 12.5 occular lens and $400 \mathrm{~mm}$ objective lens with $25 \mathrm{X}$ magnification, without causing stress on the vocal cords. The procedure involves placing the microlaryngoscope between the upper and lower teeth, over the tongue and down the throat to allow a good view of the larynx and vocal cords. The microlaryngoscope is a hollow metal tube with a fiber optic light. There was no surgical intervention aimed at altering the larynx, irrespective of vocal cord examination findings. All laryngeal examinations were video recorded for later reassessment. The sulcus vocalis were described according to Ford et al (1996). ${ }^{5}$ 
All the participants underwent the following voice evaluatiosymptoms scale (VSS), to ensure that all the participants were asymptomatic; auditory perception evaluation of the voice, with vocal assessment using the grade, roughness, breathiness, asthenia, strain, instability (GRBASI) scale; and acoustic evaluation of the voice applying the VOXMETRIA program (CTS Informática, Pato Branco, Paraná, Brazil). A sample characterization questionnaire was conducted before the vocal assessment. All the procedures were performed a minimum of 15 days after surgery.

The VSS is an instrument adapted and validated for Brazil. $^{16}$ It self-assesses voice and vocal symptoms, via 30 questions divided into three domains, collecting information on functionality (15 questions), emotional impact (8 questions) and physical symptoms (7 questions) that a voice disorder can cause. Subjects responded individually to the scale questions and each answer was scored from 0 to 4 , according to the frequency reported: (0) never, (1) rarely, (2) sometimes, (3) almost always, (4) always. The scores were used to determine the participants' level of vocal alteration.

For auditory perception and vocal acoustics, samples of the subjects' voices were recorded. All the tasks were executed with the patients comfortably seated in a quiet room, where voices were recorded individually. The vocal data for auditory perception and acoustic analysis were recorded using the Fonoview (CTS Informática) and Voxmetria software (CTS Informática), respectively, in an HP Intel Core i5 $2.5 \mathrm{GHz} 4096 \mathrm{MB}$ laptop. The voices were captured with a Karsect HT-9 microphone placed four centimeters from the speaker's mouth at a $45^{\circ}$ angle. In addition, an Andrea PureAudio USB adaptor was connected to the laptop to reduce background noise.

For auditory perception data collection, the tasks selected were sustained emission of the vowels / $/ \mathrm{a}$ and /i/ and counting from one to ten. To determine vocal parameters, the GRBASI scale, proposed by Hirano (1981) and complemented with Dejonckere's "I" parameter (1996) was applied. This scale analyzes the following aspects of vocal quality: voice roughness $(\mathrm{R})$, breathlessness $(\mathrm{B})$, asthenia $(\mathrm{A})$, stress $(\mathrm{S})$ and instability $(\mathrm{I})$, which, taken together, determine the overall degree of hoarseness $(G)$. Each of these aspects can be classified on a severity scale from 0 to 3, where 0 represents no change; 1 slightly changed; 2 moderately changed and 3 significantly changed.

Auditory perception of voices was evaluated by two speech therapists specialized in voice assessment, with more than 15 years' experience. To determine inter-rater agreement, $30 \%$ of the voices were randomly repeated for a total of 34 voices. The results obtained from the evaluator with the highest index of reliability were selected for analysis.

For acoustic recording of the voices of the participants using the Voxmetria (CTS Informática) software, subjects were instructed to emit the vowel /e/ and count from 1 to 10 . The following parameters were investigated: a) fundamental frequency; b) vocal intensity; c) irregularity d) jitter; e) shimmer; and f) glottal noise excitation ratio (GNE). The acoustic data were supplied by the program itself.

Vocal self-assessment, auditory perception and the acoustic data of groups 1 and 2 were submitted to descriptive analysis using absolute and percentage frequencies for the categorical variables and means and standard deviation for the numerical variables.

Pearson chi-squared or Fisher Exact test were applied to determine whether there was an intergroup difference in the categoriacal variables and the Student $t$ or Mann-Whitney test to compare the numerical variables. Fisher Exact test rather than Pearson chi-squared was used when the condition to apply the latter was not present. The Student $t$-test was selected when the hypothesis of data normality was confirmed in both groups, while the Mann-Whitney test was used when the data were not normally distributed. Data normality was determined by the Shapiro-Wilk test and equality of variance by Levene test.

Cohen Kappa Statistic was used to analyze inter-rater agreement, considering the classification proposed by Landis \& Koch, as follows: (a): almost perfect: kappa between 0.80 and 1.00; (b): substantial: kappa between 0.60 and 0.80; (c): moderate: kappa between 0.40 and 0.60 ; (d): fair: kappa betweeen 0.20 and 0.40 ; (e): slight: kappa between zero and 0.20 ; (f): poor: kappa between -1 and zero. The margin of error used in the statistical tests was $5 \%$. The data were entered into an Excel (Microsoft Corp. Redmond, WA, USA) spreadsheet and the Statistical Package for the Social Sciences (SPSS) version 21 (IBM Corp., Armonk, NY, USA) was used to obtain the statistical calculations.

\section{Results}

The age of the participants varied between 24 and 66 years, with an average of 41.88 years. Half were aged between 24 and 40 years and the other half between 41 and 66 years; a majority (69.2\%) were women. Only two individuals (7\%) were voice professionals, one each from group 1 and 2 .

Group 1 consisted of individuals with sulcus vocalis and group 2 without the disorder. Of the 26 vocal cords studied in group 1,23 exhibited sulci vocalis. Of these, 18 (78\%) were type I and 5 (22\%) type II. Furthermore, most of the individuals with sulcus vocalis (77\%) were affected bilaterally $(n=10)$.

- Table 1 illustrates the mean VSS values, and the standard deviation. There was no statistically significant intergroup difference $(p>0.05)$. The values observed for each individual were below 16 points, when all the domains were added. This assessment ensured that none of the participants displayed vocal symptoms.

- Table 2 shows the auditory perception results obtained, considering each parameter on the GRBASI scale, according

Table 1 Means and standard deviations of the vocal symptoms scale values obtained

\begin{tabular}{|l|l|l|l|}
\hline Variable & $\begin{array}{l}\text { Group 1 Mean } \\
\text { (standard } \\
\text { deviation) }\end{array}$ & $\begin{array}{l}\text { Group 2 Mean } \\
\text { (standard } \\
\text { deviation) }\end{array}$ & $P$-value \\
\hline • VSS & $6.38(2.29)$ & $8.46(3.77)$ & $0.148^{*}$ \\
\hline
\end{tabular}

Abbreviation: VSS, vocal symptoms scale.

*Mann-Whitney test. 
e334 Laryngeal and Vocal Characterization Soares et al.

Table 2 Numerical and percentage data of group 1 and 2 subjects with normal and altered auditory perception vocal parameters

\begin{tabular}{|c|c|c|c|c|c|c|c|}
\hline \multirow{3}{*}{ Variable } & \multicolumn{4}{|c|}{ Group } & & & \multirow{3}{*}{$P$-value } \\
\hline & \multicolumn{2}{|c|}{ Group 1} & \multicolumn{2}{|c|}{ Group 2} & \multicolumn{2}{|c|}{ Group Total } & \\
\hline & $N$ & $\%$ & $N$ & $\%$ & $N$ & $\%$ & \\
\hline TOTAL & 13 & 100.0 & 13 & 100.0 & 26 & 100.0 & \\
\hline \multicolumn{8}{|l|}{$\cdot G$} \\
\hline Normal & 4 & 30.8 & 11 & 84.6 & 15 & 57.7 & $p^{\mathrm{a}}=0.005^{*}$ \\
\hline Overall & 9 & 69.2 & 2 & 15.4 & 11 & 42.3 & \\
\hline \multicolumn{8}{|l|}{ - $\mathrm{R}$} \\
\hline Normal & 5 & 38.5 & 11 & 84.6 & 16 & 61.5 & $p^{a}=0.016$ \\
\hline Overall & 8 & 61.5 & 2 & 15.4 & 10 & 38.5 & \\
\hline \multicolumn{8}{|l|}{ - B } \\
\hline Normal & 12 & 92.3 & 13 & 100.0 & 25 & 96.2 & $p^{\mathrm{b}}=1,000$ \\
\hline Overall & 1 & 7.7 & - & - & 1 & 3.8 & \\
\hline \multicolumn{8}{|l|}{ - $A$} \\
\hline Normal & 13 & 100.0 & 13 & 100.0 & 26 & 100.0 & ${ }^{* *}$ \\
\hline Overall & - & - & - & - & - & - & \\
\hline \multicolumn{8}{|l|}{$\cdot 5$} \\
\hline Normal & 12 & 92.3 & 12 & 92.3 & 24 & 92.3 & $p^{\mathrm{b}}=1,000$ \\
\hline Overall & 1 & 7.7 & 1 & 7.7 & 2 & 7.7 & \\
\hline \multicolumn{8}{|l|}{$\cdot 1$} \\
\hline Normal & 13 & 100.0 & 13 & 100.0 & 26 & 100.0 & ** \\
\hline Overall & - & - & - & - & - & - & \\
\hline
\end{tabular}

*Significant difference at the $5.0 \%$ level.

${ }^{* *}$ Could not be determined due to the presence of only one category.

${ }^{a}$ Through Pearson Chi-square test.

busing Fisher exact test.

to the group analyzed. In group $1,69.2 \%$ of the individuals displayed mild overall vocal alteration (G), and $61.5 \%$ mild roughness (R). In group 2, 15.4\% showed mild overall vocal alteration $(G)$ and roughness (R). Intergroup comparison revealed a significant difference $(p<0.05)$ in relation to the overall degree of vocal alteration $(G)$ and roughness $(R)$.

The weighted kappa index values of inter-rater agreement analysis on the GRBASI auditory perception scale were 0.81 (group I) and 0.82 (group II) for evaluator 1 , and 0.79 (group I) and 0.80 (group II) for evaluator 2. In this analysis, almost perfect agreement was obtained for evaluator 1 and substantial for evaluator 2, according to the Landis \& Koch classification. As such, the former's analysis was considered.

- Table 3 shows the absolute number of group 1 and 2 individuals with normal and altered values in the acoustic assessment parameters. Most of the individuals in both groups obtained normal acoustic values.

The average acoustic parameter values in - Table $\mathbf{4}$ show that the means were higher in group 1 in vocal intensity, irregularity, shimmer and GNE ratio. Mean jitter was higher in group 2. Average fundamental frequency was higher in group 1 men, while the opposite was found for women. Furthermore, mean fundamental frequency was higher in
Table 3 Numerical data of group 1 and 2 individuals with normal and altered values in the acoustic assessment parameters.

\begin{tabular}{|l|l|l|l|l|}
\hline \multirow{2}{*}{ Parameters } & \multicolumn{2}{|l|}{ Group 1 } & Group 2 \\
\cline { 2 - 5 } & Normal & Altered & Normal & Altered \\
\hline $\begin{array}{l}\text { Fundamental } \\
\text { frequency }\end{array}$ & 13 & 0 & 13 & 0 \\
\hline Intensity & 10 & 03 & 11 & 02 \\
\hline Irregularity & 10 & 03 & 11 & 02 \\
\hline Shimmer & 10 & 03 & 11 & 02 \\
\hline Jitter & 10 & 03 & 10 & 03 \\
\hline GNE ratio & 09 & 04 & 08 & 05 \\
\hline
\end{tabular}

Abbreviation: GNE, glottal-to-noise excitation.

women. However, when compared statistically, there was no significant intergroup difference $(p>0.05)$, considering all the acoustic parameters analyzed.

\section{Discussion}

Of the 13 individuals ( 26 vocal cords) with sulcus vocalis, the vast majority were type I, with type II being far less frequent. 
Table 4 Mean values and standard deviation of fundamental frequency, intensity, irregularity, shimmer, jitter and glottal-to-noise excitation ratio per group

\begin{tabular}{|l|l|l|l|l|}
\hline & \multicolumn{2}{|l|}{ Group } & & \\
\hline Variable & Group 1 & Group 2 & Group total & $P$-value \\
\hline & Mean \pm SD (Median) & Mean \pm SD (Median) & Mean \pm SD (Median) & \\
\hline • Frequency (male) & $101.48 \pm 7.44$ & $98.68 \pm 9.67$ & $100.08 \pm 8.13$ & $p^{\mathrm{b}}=0.886$ \\
\hline - Frequency (female) & $194.46 \pm 8.96$ & $202.21 \pm 17.75$ & $198.34 \pm 14.21$ & $p^{\mathrm{b}}=0.077$ \\
\hline • Intensity & $60.07 \pm 3.53(59.87)$ & $58.85 \pm 3.39(59,23)$ & $59.46 \pm 3.45(59.82)$ & $p^{\mathrm{a}}=0.337$ \\
\hline - Irregularity & $3.48 \pm 1.43(3.45)$ & $3.10 \pm 1.29(2.89)$ & $3.29 \pm 1.35(3.13)$ & $p^{\mathrm{a}}=0.485$ \\
\hline • Shimmer & $4.56 \pm 2.04(4.38)$ & $3.96 \pm 2.11(4,56)$ & $4.26 \pm 2.06(4.47)$ & $p^{\mathrm{a}}=0.469$ \\
\hline - Jitter & $0.44 \pm 0.36(0,24)$ & $0.48 \pm 0.33(0.47)$ & $0.46 \pm 0.34(0.46)$ & $p^{\mathrm{b}}=0.663$ \\
\hline - GNE ratio & $0.63 \pm 0.20(0.67)$ & $0.57 \pm 0.27(0.67)$ & $0.60 \pm 0.24(0.67)$ & $p^{\mathrm{a}}=0.580$ \\
\hline
\end{tabular}

Abbreviations: GNE, glottal-to-noise excitation; SD, standard deviation. a Through the t-Student test with equal variances.

bThrough the Mann-Whitney test.

There were no vocal cords with sulcus type III. Furthermore, most individuals with sulcus were affected bilaterally. The literature shows a predominance of bilateral sulcus, with most being type II or III. ${ }^{5,7,12-14}$ The predominance of type I sulci in the present study may be related to the fact that only individuals from the general population with no vocal complaints were selected. According to Ford, ${ }^{5}$ type I sulcus is located on the surface layer of the lamina propria, where structural damage to the mucosa is minimal, with no increase in stiffness or impact on vocal quality. By contrast, literature studies ${ }^{5,7,12-14}$ assessed individuals with voice complaints, which may explain the prevalence of type II and III sulci, resulting in greater damage to the vocal cord of the lamina propria.

In relation to vocal symptoms, none of the VSS scale values ( - Table 1) indicated hoarseness in the subjects of the present study, or significant intergroup differences. Furthermore, the total VSS value for each participant was below 16 points. As such, this population exhibited no vocal symptoms. According to Moreti et al (2014), ${ }^{16}$ who validated the VSS for Brazilian Portuguese, the cutoff point for vocal symptoms is 16 . Values greater than 16 suggest some degree of hoarseness and those below 16 no vocal symptoms.

With respect to the lack of voice-related symptoms in group 1 individuals, since most were not voice professionals and did not use strain their voice, they were less likely to overload their vocal apparatus. Moreover, the degree of required vocal quality, as well as self-perceived vocal disadvantage, tend to be lower in the general population than in voice professionals. ${ }^{17,18}$ Given that voice professionals face significant vocal demands and risks, and that even slight hoarseness can limit good performance, it is hoped that this population report sulcus vocalis-related problems as soon as possible, in contrast to the group under study.

In relation to the auditory perception characteristics of group 1, eight of the 13 individuals with sulcus vocalis exhibited mild roughness. Only one individual displayed mild breathlessness ( - Table 2 ). This result contradicts literature findings, ${ }^{5-7,10-12}$ in which breathlessness is the primary auditory perception alteration in patients with sulcus vocalis. Hirano et al (1990) ${ }^{11}$ assessed 126 patients with sulcus vocalis and most of the individuals exhibited mild breathlessness and hoarseness. The results indicated that voice quality was more correlated with glottis incompetence than vocal cord stiffness. Since it was a retrospective study of individuals with vocal complaints examined by videolaryngoscopy, the sulci detected would likely be more pronounced with a more evident vocal impact, which would explain the higher incidence of breathlessness.

Other studies of symptomatic patients also showed a predominance of breathlessness in subjects with sulcus vocalis, in addition to the presence of roughness in some cases. The authors also agree that breathlessness is related to glottis incompetence and stiffness in the lamina propria of the vocal cords. ${ }^{119}$ Bouchayer et al $(1988)^{20}$ reported that although sulcus vocalis is a benign condition, it has a dramatic impact on the voice, and the resulting vocal quality can be considered typical, not only breathlessness, but especially the rough, veiled monotone and reduced loudness, with limited harmonics and lack of projection imposed by the restricted muco-undulatory movement of the vocal cords.

Given these literature findings, contradicting those obtained here, in which individuals with sulcus vocalis exhibited mild roughness, it is important to underscore that $78 \%$ of group 1 individuals consisted of patients with type 1 sulci, which are generally shallow, exerting no significant impact on glottic closure or the voice. In addition, sulcus vocalis affects a part of the population with no vocal complaints, whereby the impact on the voice depends on the type and magnitude of the sulcus vocalis as well as the vocal demand the individual is submitted to. ${ }^{1,2,8}$ The minimal structural alteration of the larynx present since birth can manifest themselves in the first sounds an infant makes or in adulthood depending on vocal demands, irritative factors and laryngeal development itself. ${ }^{1,2,21}$

Intergroup comparison in terms of auditory perception assessment revealed that individuals with sulcus vocalis obtained lower scores, with a statistically significant difference 
in relation to group 2, in overall hoarseness $(G)$ and roughness (R), both exhibiting a mild level. The presence of roughness in group 1 individuals is related to vibratory irregularity in the mucosal wave of the vocal cords. Some authors ${ }^{1,6,11}$ report that sulcus vocalis may exhibit different levels of vibratory irregularity in the mucosal wave, when different layers of the lamina propria of the vocal cords are affected. ${ }^{4,7}$ Ushijima et al (1986) 22 considered that the sulcus vocalis is related to persistent hoarseness due to insufficient glottic closure during phonation. Other authors ${ }^{2,4,6}$ found that the impact is minimal in type 1 sulcus vocalis, because only the surface layer of the lamina propria is affected, often not perceived by the individuals who exhibit mild roughness. ${ }^{1}$

With respect to acoustic assessment, in both groups 1 and 2, all the individuals showed normal voice frequencies, with no significant intergroup differences (-Table 3 ). The normal distribution range for male voices is between 80 and $150 \mathrm{~Hz}$, while for females it varies from 150 to $250 \mathrm{~Hz} .^{1,23,24}$ Several studies found alterations in fundamental frequency in individuals with sulcus vocalis, often leading to acute frequencies. $^{6,10,25}$ However, the samples of these studies consisted of subjects with type II or Type III sulcus, likely with vocal cords that exhibited important structural alterations. In the present study, most of the sulci were type I, with minimal structural alteration of the mucosa and no increase in stiffness or impact on the fundamental frequency. These results corroborate those reported by Lim et al (2009), ${ }^{6}$ who assessed individuals with type I sulcus vocalis and found normal frequency values.

Intensity is directly related to subglottic pressure of the air column, which depends on factors such as amplitude of the vibration and stress on the vocal cords, more specifically glottic resistance. ${ }^{1,26}$ With respect to this parameter, normal mean values were observed for both groups, with no significant differences between them (-Table 4). Considering that individuals in the present study with sulcus vocalis exhibited minimal structural alterations to their vocal cords with no impact on glottic closure, no change in subglottic air pressure or voice intensity is expected.

The parameter irregularity is related to glottic coaptation and quantifies irregularity of vocal cord vibratory cycles. ${ }^{1,27}$ In the present study, the average values were normal for both groups, with no significant differences between them.

In relation to shimmer, the individuals with sulci vocalis displayed normal values and no significant intergroup differences were observed. Shimmer indicates the variability in sound wave amplitude, that is, irregular alterations in the amplitude of glottic cycles, from one to another. ${ }^{1,5,11}$ Since the individuals studied here likely showed no decline in glottic resistance or mass lesion, according to the literature, ${ }^{1,28}$ they may experience alterations in shimmer values, thereby explaining the fact that most of the values were normal.

In regard to jitter, the means obtained in groups 1 and 2 were normal. Jitter showed variable fundamental frequency and how much a period is different from its predecessor or immediate successor. Furthermore, alterations occur due to the lack of vocal cord vibration control, often correlated with roughness. ${ }^{1,5,11}$ The results of the present study demonstrated that the changes found did not cause an increase in vocal cord vibration periodicity, which is reflected in higher jitter values. ${ }^{1}$ The type of sulcus found does not result in significant damage to the vocal cord mucosa.

With respect to the jitter and shimmer data obtained in this study, it is important to underscore the findings of Yilmaz, ${ }^{15}$ who studied the acoustic data of 44 patients with sulcus vocalis. In his study, patients exhibited changes in several acoustic parameters (jitter, shimmer, and fundamental frequency), without characterizing the type of sulcus vocalis. It is important to emphasize, however, that since this study assessed a sulcus excision technique associated with vocal cord medicalization, the population analyzed displayed intense vocal complaints and sulci vocalis with greater structural vocal cord alterations. ${ }^{29-31}$

Glottal-to-noise excitation is an acoustic measure that calculates the noise produced by vocal cord oscillation, indicating that the vocal signal originates in vocal cord vibrations or the turbulent air current produced in the vocal tract. $^{32}$ The mean GNE obtained in group 1 and 2 revealed normal values, with no significant intergroup differences. According to the literature, since the GNE is related to breathlessness, the normal average values can be explained, since roughness was the only parameter that stood out in the individuals assessed (predominant in group 1). According to Madazio et al (2009), ${ }^{33}$ strained and adapted voices may exhibit normal mean GNE values.

In regard to the difference in auditory perception and acoustic data, assessment of the former considers both source and filter-related data, which could, in some situations, change the overall impression of voice. It is known that correlations between auditory perception and acoustic data do not always exist. ${ }^{1}$

According to Pontes, ${ }^{2}$ a larynx with sulcus vocalis or other minimal structural alterations (MSA) can remain balanced and adapted to the vocal demand of speakers without compromising them for the rest of their life. The slight vocal alterations observed in individuals with sulcus vocalis reinforce the hypothesis that its vocal impact may be minimal or nonexistent, but these slight vocal deviations can be detected by voice specialists. Therefore, alterations in the vocal quality of individuals with sulcus is far greater than that traditionally described in the literature, since, in addition to symptomatic patients with pronounced sulci and severe hoarseness, where roughness and breathlessness predominate, there is also a much larger population of asymptomatic individuals with sulcus stria minor that may display minimum alterations in voice quality. ${ }^{34}$ This sample clearly demonstrates the possibility of classifying one of the most frequent MSAs (sulcus vocalis) as an anatomical variation (morphological alteration in which the function of the organ is not compromised) or a representative entity of the set of laryngeal abnormalities, since in certain situations, this lesion promotes phonatory deviation.

\section{Conclusion}

Type I sulcus vocalis is predominant in individuals with no voice complaints, who may exhibit slight changes in vocal 
quality, characterized by roughness, or no voice alterations whatsoever.

\section{Conflicts of Interest}

The authors declare that there were no conflicts of interest.

\section{References}

1 Behlau M, Azevedo R, Pontes P. Concept of normal voice and classification of dysphonias. In: Behlau M, editor. Voice: The Specialist's Book. 2a ed. Vol.1. Rio de Janeiro: Revinter; 2004:53-79

2 Pontes P, Behlau M, Gonçalves I. Alterações estruturais mínimas da laringe (AEM): considerações básicas. Acta AWHO. 1994;13:2-6

3 Hirano M. Morphological structure of the vocal cord as a vibrator and its variations. Folia Phoniatr (Basel) 1974;26(02):89-94

4 Nakayama M, Ford CN, Brandenburg JH, Bless DM. Sulcus vocalis in laryngeal cancer: a histopathologic study. Laryngoscope 1994; 104(1 Pt 1):16-24

5 Ford CN, Inagi K, Khidr A, Bless DM, Gilchrist KW. Sulcus vocalis: a rational analytical approach to diagnosis and management. Ann Otol Rhinol Laryngol 1996;105(03):189-200

6 Lim JY, Kim J, Choi SH, et al. Sulcus configurations of vocal folds during phonation. Acta Otolaryngol 2009;129(10):1127-1135

7 Hsiung MW, Woo P, Wang HW, Su WY. A clinical classification and histopathological study of sulcus vocalis. Eur Arch Otorhinolaryngol 2000;257(08):466-468

8 Pontes P, Ramos HVL. Alterações estruturais mínimas. In: Ganança FF, Pontes P, editores. Manual de Otorrinolaringologia e Cirurgia de Cabeça e Pescoço. 1a ed. Barueri: Manole; 2011:25-38

9 Akbulut S, Altintas H, Oguz H. Videolaryngostroboscopy versus microlaryngoscopy for the diagnosis of benign vocal cord lesions: a prospective clinical study. Eur Arch Otorhinolaryngol 2015;272 (01):131-136

10 Pontes P, Behlau M. Sulcus mucosal slicing technique. Curr Opin Otolaryngol Head Neck Surg 2010;18(06):512-520

11 Hirano M, Yoshida T, Tanaka S, Hibi S. Sulcus vocalis: functional aspects. Ann Otol Rhinol Laryngol 1990;99(9 Pt 1):679-683

12 Oliveira CJ, Gama ACC, Oliveira JB, Neto ALR. Acoustical analysis and perceptual ratings of voice before and after surgery for implantation of pre-fascia temporal muscle. Rev CEFAC 2008;10:76-83

13 Grellet M, Carneiro CG, Aguiar LN, Rosa MO, Pereira JC. The pediculated flap technique to sulcus vocalis repairing. Rev Bras Otorrinolaringol 2002;68:75-79

14 Macedo Filho ED, Caldart AU, Assis C, Pletsch F, Mocellin M. Internal Section of the Vocal Ligament - A New Technique for the Treatment of the Sulcus Vocalis Arq. Int. Otorrinolaringol. 2007; 11:254-259

15 Yllmaz T. Sulcus vocalis: excision, primary suture and medialization laryngoplasty: personal experience with 44 cases. Eur Arch Otorhinolaryngol 2012;269(11):2381-2389

16 Moreti F, Zambon F, Oliveira G, Behlau M. Cross-cultural adaptation, validation, and cutoff values of the Brazilian version of the Voice Symptom Scale-VoiSS. J Voice 2014;28(04):458-468
17 Chun RYS, Servilha EAM, Santos LMA, Sanches MH. Health Promotion: the student's knowledge of journalism about his voice. Distúrb Comun 2007;19:73-80

18 Aquino FS, Teles LCS. Vocal self-perception of professional singers. Rev CEFAC 2013;15:986-993

19 Vieira VP, Biase N, Pontes P. Acoustical and Perceptive Auditive Analysis Verses Glottic Coaptation in Minimal Structural Alteration. Acta ORL. 2006;24:174-180

20 Bouchayer M, Cornut G. Microsurgery for benign lesions of the vocal folds. Ear Nose Throat J 1988;67(06):446-449, 452-454, 456-464 passim

21 Silva AR, Machado AJ Jr, Crespo AN. Anatomical study of minor alterations in neonate vocal folds. Rev Bras Otorrinolaringol (Engl Ed) $2014 ; 80(04): 311-317$

22 Ushijima T, Kase Y, Honda K, Niimi S, Hirose H. Effectiveness of intracordal injection for sulcus vocalis. Folia Phoniatr (Basel) 1986;38:365-368

23 Araujo SA, Grellet M, Pereira JC. Standardization of acoustic measures of the normal voice. Rev Bras Otorrinolaringol 2002; 68:540-544

24 Naufel de Felippe AC, Grillo MHMM, Grechi TH. Standardization of acoustic measures for normal voice patterns. Rev Bras Otorrinolaringol (Engl Ed) 2006;72(05):659-664

25 Pinho SMR. Assessment and treatment of voice. In: Pinho SMR, editor. Fundamentals in Speech-Language Pathology Treating Voice Disorders. Rio de Janeiro: Guanabara Koogan; 1998:1-37

26 Wertzner HF, Schreiber S, Amaro L. Analysis of the fundamental frequency, jitter, shimmer and vocal intensity in phonological disordered children. Rev Bras Otorrinolaringol (Engl Ed) 2005; 71:582-588

27 Sanchez RF. Nonlinear acoustic voice analysis after partial laryngectomy [doctoral thesis]. São Paulo (SP): Federal University of São Paulo; 2014

28 Navas DM. Computerized analysis of fundamental frequency and its variations in height (jitter) and intensity (shimmer) of voices of children of the city of São Paulo. Pro Fono 1989;1:17-22

29 Verma P, Pal M, Raj A. Objective acoustic analysis of voice improvement after phonosurgery. Indian J Otolaryngol Head Neck Surg 2010;62(02):131-137

30 Thomas G, Mathews SS, Chrysolyte SB, Rupa V. Outcome analysis of benign vocal cord lesions by videostroboscopy, acoustic analysis and voice handicap index. Indian J Otolaryngol Head Neck Surg 2007;59(04):336-340

31 Johns MM, Garrett CG, Hwang J, Ossoff RH, Courey MS. Quality-oflife outcomes following laryngeal endoscopic surgery for nonneoplastic vocal fold lesions. Ann Otol Rhinol Laryngol 2004;113 (08):597-601

32 Michaelis D, Gramss T, Strube HW. Glottal-to-noise excitation ratio - a new measure for describing pathological voices. Acustica 1997;83:700-706

33 Madazio G, Leão S, Behlau M. The phonatory deviation diagram: a novel objective measurement of vocal function. Folia Phoniatr Logop 2011;63(06):305-311

34 Moraes BT. Prevalência das alterações estruturais mínimas da laringe: influência sobre o conceito de prega vocal normal [tese]. São Paulo (SP): Universidade Federal de São Paulo; 2015 